\title{
Fitting homocysteine to disease models, as well as adjusting the models to the disease
}

\author{
Friedrich C. Luft $^{1}$
}

Published online: 9 May 2015

(C) Springer-Verlag Berlin Heidelberg 2015

Homocysteine is a nonprotein $\alpha$-amino acid and differs from cysteine by having an additional methylene bridge. Homocysteine is not present in the diet, and all homocysteine in the body is biosynthesized from the essential amino acid methionine. Homocysteine is a product of methionine demethylation and is recycled into methionine or alternatively converted into cysteine with the assistance of B vitamins. Homocysteine is metabolized through two pathways, namely remethylation and transsulfuration. Much information has been generated from mouse models. A summary of this model information, as presented by Dayal and Lentz [1], is shown (Fig. 1). The remethylation pathway requires vitamin $\mathrm{B}_{12}$, folate, and the enzyme 5,10-methylene-tetrahydrofolate reductase (MTHFR). In the kidney and liver, homocysteine is also remethylated by the enzyme betaine homocysteine methyltransferase (BHMT), which transfers a methyl group to homocysteine via the demethylation of betaine to dimethylglycine (DMG). The transsulfuration pathway requires the enzyme cystathionine beta-synthase (CBS) and vitamin $\mathrm{B}_{6}$ (pyridoxal-5'-phosphate). Once formed from cystathionine, cysteine can be utilized in protein synthesis and glutathione (GSH) production.

Genetics has contributed greatly to our understanding of homocysteine, its metabolism, and cardiovascular risk. Classical homocystinuria, an autosomal-recessive disease, is caused by CBS deficiency. The defect leads to a multisystem disorder involving connective tissue, muscle, the central nervous system, and the cardiovascular system [2]. Genetic var-

Friedrich C. Luft

luft@charite.de

1 Experimental and Clinical Research Center, Charité Medical Faculty and the Max Delbrück Center for Molecular Medicine,

Lindenbergerweg 80, 13125 Berlin, Germany iation in the MTHFR gene influences the susceptibility to occlusive vascular disease, neural tube defects, Alzheimer's disease and other dementias, colon cancer, and acute leukemia [2]. Thus, homocysteine, its metabolism, and the enzymes involved are of great interest in biomedical research.

Homocysteine contributes to endothelial injury and subsequent atherosclerosis and is a specific risk factor for cardiovascular disease. As a result, much effort has been expended in clinical trials for various vascular diseases in the hopes that by reducing homocysteine levels, cardiovascular endpoints could be ameliorated. For instance, an updated Cochrane review found no evidence to suggest that homocysteinelowering interventions in the form of supplements of vitamins $\mathrm{B}_{6}, \mathrm{~B}_{9}$, or $\mathrm{B}_{12}$ given alone or in combination should be used for preventing cardiovascular events [3, 4]. Neither has the supplementation of dietary folic acid to lower homocysteine levels led to more favorable outcomes $[5,6]$. Nevertheless, folic acid supplementation has a major impact on reducing neural tube defects [7].

$J \mathrm{Mol} \mathrm{Med} \mathrm{this} \mathrm{month} \mathrm{features} \mathrm{a} \mathrm{homocysteine-based} \mathrm{hy-}$ pothesis in a complicated animal model. Muthuramu et al. point out that a "causal role of homocysteine in myocardial biology and function cannot be proven since residual confounding in multivariable models may occur" [8]. How very true! They studied $\mathrm{Cbs}$ heterozygous gene-deleted $(\mathrm{Cbs}+/-)$ mice. These mice were crossed with low-density lipoprotein receptor $(\mathrm{Ldl} r-/-)$ gene-deleted mice to generate $\mathrm{Cbs}+/-/$ $L d l r-/-$ mice. The mice were divided into three groups and fed regular chow, folic acid-depleted chow, or methionineenriched chow. Three weeks after diet initiation, the mice were intravenously injected with a hepatocyte-specific adenoviral vector expressing Cbs (AdCBS), with the same dose of control vector, or with saline buffer. Two weeks after that, the mice were subjected to thoracic aortic constriction (TAC), an experimental aortic coarctation, or sham operation to induce 


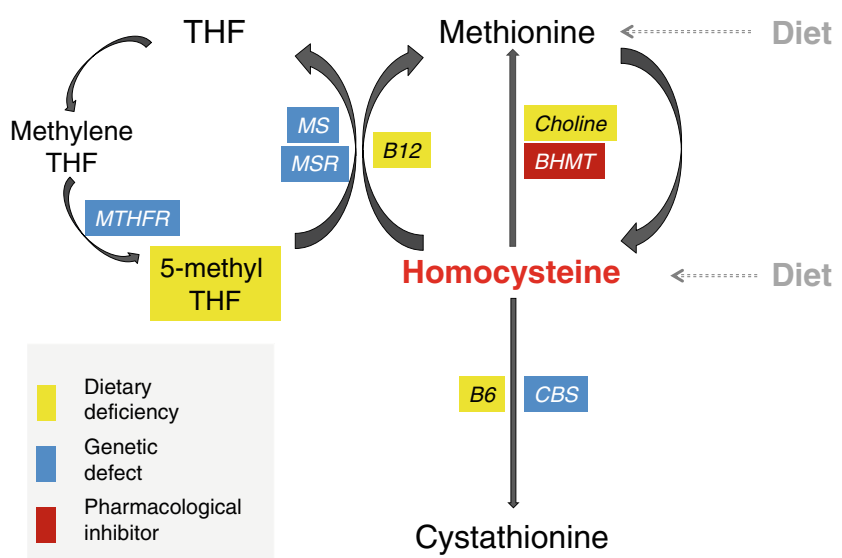

Fig. 1 Homocysteine is an intermediate metabolite of the methionine cycle. Homocysteine can be metabolized to cystathionine by cystathionine beta-cystathionine synthase (CBS), requiring the cofactor vitamin $\mathrm{B}_{6}$. Homocysteine can also be remethylated to methionine by methionine synthase (MS) with vitamin $B_{12}$. This reaction uses a methyl group from 5-methyl tetrahydrofolate (THF) and links the methionine cycle with the folate cycle. The activity of methylene tetrahydrofolate reductase (MTHFR) derives 5-methyl THF. Methionine synthase reductase (MSR) is required to maintain MS in its active conformation. The liver and kidney homocysteine can be remethylated to methionine by betaine/homocysteine methyltransferase (BHMT). This reaction uses betaine, which is derived from choline, as a methyl donor. Hyperhomocysteinemia can be induced in mice by administering methionine or homocysteine, dietary deficiency of folate, vitamin $\mathrm{B}_{6}$, vitamin $\mathrm{B}_{12}$, or choline (yellow); by a genetic deficiency of CBS (as in the current study), MTHF $\mathrm{R}, \mathrm{MS}$, or MSR (blue); or by pharmacologically inhibiting BHMT (red). Scheme adapted from Dayal and Lentz [1]

heart disease. Adenoviral transfer lowered homocysteine values in TAC mice and sham controls. Hemodynamic measurements were performed with a Millar pressure catheter. In short, replacing the CBS function made the mice better. Survival was improved after TAC, compared to untreated mice, and the end-diastolic pressure was reduced to almost sham values and reflectors of diastolic function were improved. The authors monitored antioxidant defense mechanisms and found that replacing CBS function reduced myocardial oxidative stress.

The animal model, complex and confounded as it is, is convincing. However, what do we carry away that is really new here? More than 200 articles in the literature underscore the relationship between hyperhomocysteinemia and heart failure. More than 1000 papers are concerned with hyperhomocysteinemia and increased oxidative stress. Viral gene transfer of $C b s$ to replace $C b s$ in $C b s$-heterozygous genedeficient mice is a nice touch and eliminates variables imposed by dietary manipulation. However, the results were largely predictable. Will we encounter patients with $C B S$ deficiency that simultaneously have familial hypercholesterolemia and then happen to have developed coarctation of the aorta? The studies underscore what must be done to mice to make them sick; the relevance to any human disease is uncertain.
Actually, MFTR polymorphisms have been studied in patients with familial hypercholesterolemia $(L D L R+/-)$. Indeed, the homocysteine levels in patients with the risk variant (TT) were higher and their high-density lipoprotein (HDL) cholesterol levels were lower than persons not carrying this MFTR variant [9]. The mechanism of interaction between plasma homocysteine and lipid metabolism is unknown. Interestingly, coarctation of the aorta has also been associated with MFTR the TT polymorphism [10], so perhaps the authors' model is not so far-fetched after all.

In terms of "translational research," the fact that all the intervention trials directed at reducing homocysteine have invariably failed is certainly disappointing. However, perhaps the human conditions have very little to do with the animal studies. Thus, it is not clear whether or not homocysteine actually plays a causal role in many diseases with which it is associated. An alternative possibility could be that homocysteine is instead a marker for some other underlying mechanism. A large body of data links hyperhomocysteinemia and folate status with oxidant stress. The authors of the current studies also made oxidative stress their focus. Hoffmann recently reviewed data suggesting that homocysteine not only promotes cellular and protein injury via oxidant mechanisms but also serves as a marker for the presence of pathological oxidant stress [11]. Of course, the antioxidant trials to reduce cardiovascular disease were as disappointing as the homocysteine trials [12]. Humans just seem to be a stubborn, outbred, heterogeneous animal model that has little utility in explaining diseases in rodents.

Respectfully,

Friedrich C. Luft

\section{References}

1. Dayal S, Lentz SR (2008) Murine models of hyperhomocysteinemia and their vascular phenotypes. Arterioscler Thromb Vasc Biol 28(9):1596-1605

2. Fodinger M, Horl WH, Sunder-Plassmann G (2000) Molecular biology of 5,10-methylenetetrahydrofolate reductase. J Nephrol 13(1):20-33

3. Marti-Carvajal AJ, Sola I, Lathyris D, Karakitsiou DE, SimancasRacines D (2013) Homocysteine-lowering interventions for preventing cardiovascular events. Cochrane Database Syst Rev 1: CD006612

4. Marti-Carvajal AJ, Sola I, Lathyris D (2015) Homocysteinelowering interventions for preventing cardiovascular events. Cochrane Database Syst Rev 1:CD006612

5. Marti-Carvajal AJ, Sola I, Lathyris D, Salanti G (2009) Homocysteine lowering interventions for preventing cardiovascular events. Cochrane Database Syst Rev 4:CD006612

6. Mei W, Rong Y, Jinming L, Yongjun L, Hui Z (2010) Effect of homocysteine interventions on the risk of cardiocerebrovascular events: a meta-analysis of randomised controlled trials. Int J Clin Pract 64(2):208-215 
7. Blencowe H, Cousens S, Modell B, Lawn J (2010) Folic acid to reduce neonatal mortality from neural tube disorders. Int $\mathrm{J}$ Epidemiol 39(Suppl 1):i110-i121

8. Muthuramu I, Singh N, Amin R, Nefyodova E, Debasse M, Horenbeeck IV, Jacobs F, De Geest B (2015) Selective homocyseine-lowering gene transfer attenuates pressure-overloadinduced cardiomyopathy via reduced oxidative stress. J Mol Med. doi:10.1007/s00109-015-1281-3

9. Real JT, Martinez-Hervas S, Garcia-Garcia AB, Chaves FJ, Civera M, Ascaso JF, Carmena R (2009) Association of C677T polymorphism in MTHFR gene, high homocysteine and low HDL cholesterol plasma values in heterozygous familial hypercholesterolemia. J Atheroscler Thromb 16(6):815-820

10. Kuehl K, Loffredo C, Lammer EJ, Iovannisci DM, Shaw GM (2010) Association of congenital cardiovascular malformations with 33 single nucleotide polymorphisms of selected cardiovascular disease-related genes. Birth Defects Res A Clin Mol Teratol 88(2):101-110

11. Hoffman M (2011) Hypothesis: hyperhomocysteinemia is an indicator of oxidant stress. Med Hypotheses 77(6):1088-1093

12. Leopold JA (2015) Antioxidants and coronary artery disease: from pathophysiology to preventive therapy. Coron Artery Dis 26(2): 176-183 